\title{
LA ETNOEDUCACIÓN DE LA REFLEXIÓN A LA ACCIÓN
}

\author{
Claribel Ochoa Romero ${ }^{1}$ \\ Alexis Carabali Angola²
}

\begin{abstract}
Resumen
La etnoeducación como propuesta universitaria constituyó un importante paso en el reconocimiento de los contextos étnicos y sus derechos a la protección de su lengua, cultura y territorio. La realidad indígena latinoamericana planteó similares problemáticas de los indígenas, la reforma constitucional de 1991, en Colombia posibilitó la puesta en marcha, desde la Universidad de La Guajira del primer programa en etnoeducación que profesionalizó a los profesores indígenas del departamento. Entre la legislación tendiente a la especialización de los docentes y la carencia de propuestas articuladoras de las diversas iniciativas esta iniciativa terminó siendo un enfoque educativo circunscrito a las etnias y no una solución educativa para la diversidad étnica nacional.
\end{abstract}

Palabras clave: etnoeducación, etnodesarrollo, diversidad étnica, wayuu, universidad de la Guajira.

1 Docente Investigadora Grupo Territorio Semiáridos del Caribe, Universidad de La Guajira. Antropóloga, Universidad del Cauca. Especialista en Evaluación Escolar. Magister en Ciencias de la Educación. Correo: clarigua@hotmail.com

2 Docente Investigador Grupo Territorio Semiáridos del Caribe, Universidad de La Guajira. Antropólogo, Universidad del Cauca. Especialista en Historia de América, Universidad del Valle. Magister en Estudios del Cribe, Universidad Nacional de Colombia, sede San Andrés. Doctor en Antropología, Universidad de los Andes de Mérida, Venezuela. Correo: Alcaanster@gmail. com

DOI: $10.21892 / 9789585547971.6$ 


\begin{abstract}
Ethno-education as a university proposal was an important step in the recognition of ethnic contexts and their rights to the protection of their language, culture and territory. The indigenous reality of Latin America raised similar problems of the indigenous, the constitutional reform of 1991, in Colombia made possible the start, from the University of La Guajira of the first program in ethnoeducation that professionalized the indigenous teachers of the department. Among the legislation aimed at the specialization of teachers and the lack of proposals articulating the various initiatives this initiative ended up being an educational approach circumscribed to ethnic groups and not an educational solution for national ethnic diversity.
\end{abstract}

Keywords: ethnoeducation, ethnodevelopment, ethnic diversity, wayuu, University of La Guajira. 


\section{Introducción}

Según Burchardt (2008, p. 9-10):

En América Latina la fuerte articulación sostenida entre la desigualdad socio económica y las posibilidades volátiles de participación social de los grupos más desaventajados en la vida colectiva e institucional, dan cuenta de una tendencia profunda a la perpetuación de la exclusión, el desempleo, la inseguridad, la marginación educativa, la ilegalidad y el riesgo que afecta primordialmente a los grupos que pertenecen a "minorías" étnico-culturales (indígenas y afrolatinos). Estas problemáticas se tornan realmente preocupantes si se considera el gran número de personas excluidas por motivos socio-culturales en toda la región. En América Latina y el Caribe existen hasta 40 millones de indígenas distribuidos en más de 400 grupos étnicos. El 90\% de la población indígena latinoamericana se concentra en Perú (27\%), México (26\%), Guatemala (15\%), Bolivia (12\%) y Ecuador (8\%). Dentro de las respectivas sociedades las poblaciones indígenas constituyen una gran parte de la población: Bolivia (67\%), Perú (45\%), Guatemala (40\%), México (30\%) y Ecuador (20\%). Por su parte, la población afro-descendiente alcanza aproximadamente a 150 millones de personas, lo que es proporcional al 30\% de la población total de la región. Considerando su distribución geográfica, la misma se reparte del siguiente modo: Brasil (50\%), Colombia (20\%) y Venezuela (10\%) entre otros. Dentro de los países, las poblaciones afro - latinas, en relación a la población total, constituyen en Brasil (50\% entre pardos y negros), Colombia (15\% entre mulatos y negros) y Venezuela (10\%). Muchas de las mencionadas comunidades culturales poseen idiomas propios al 
igual que cosmovisiones y sistemas políticoeconómicos enraizados en las propiedades de su entorno natural y social. Trágicamente, en muy pocos casos, esta variedad cultural ha sido integrada en la concepción de los Estados latinoamericanos.

Ladiversidad cultural vista desdela propuesta del multiculturalismo plantea un camino que asegure la participación de la diversidad étnica en la nación colombiana y en particular en el departamento de La Guajira. De acuerdo con esto nos dice Tylor (2000, p. 170). "Los movimientos de emancipación en las sociedades multiculturales no configuran un fenómeno unitario". Es este el caso de la emergencia de los afro y sus reclamos, además de lo indígena como diferenciado, no es igual un indígena del caribe que uno de los andes o de la Amazonía, igual situación se presenta para los afrodescendientes.

Krehof (2000, p. 74), habla de la otredad desigual:

Hay, por un lado, una cultura a plenitud, que es la occidental que conocemos en el caso nuestro y, por otro, culturas subalternas que sobreviven y se reproducen por fragmentos, cuya característica principal es que son culturas que no tienen poder, que no ejercen poder alguno para imponer sus ideas, difundir sus conceptos y sus propios valores. La política se hace sin ellos y contra ellos, sin ellas y contra ellas. 


\section{Antecedentes}

\section{Educación Indígena y el Concepto de Etnoeducación}

Cada cambio económico propicia una política educativa, con un pensamiento pedagógico para la población en general, que promueve sus intereses. Para el caso de las comunidades indígenas, excluidas de la escolaridad formal y del sistema productivo capitalista, ésta estuvo adscrita al dominio de la iglesia católica que orientó toda su enseñanza en asimilarlos a la cultura occidental en el plano ideológico y no productivo.

Según Ahern (1991, p. 13), en 1820 Francisco de Paula Santander ordenó que se estableciera una escuela en toda comunidad de más de 30 familias, además consideró que los niños indígenas debían ser educados en las mismas condiciones que los blancos. Para GómezMartínez (2004, p. 6), en 1825 Bolívar considera que:

Los pobres indígenas se hallan en un estado de abatimiento verdaderamente lamentable. Yo pienso hacerles todo el bien posible: primero, por el bien de la humanidad, y segundo, porque tienen derecho a ello, y últimamente, porque hacer bien no cuesta nada y vale mucho. Y, en efecto, el 4 de julio de 1825, promulga un decreto proclamando los derechos del "indio" y prohibiendo su explotación.

Durante la mayor parte del siglo XIX la educación indígena fue contenida en esos dictámenes legales hasta la declaración de la constitución de 1886 que como se verá más adelante, nos da una idea del factor predominante de las leyes que rigieron la educación en Colombia desde los inicios de la era republicana como manifiesta Bodnar (1994), la educación oficial para comunidades indígenas obedecía al Concordato entre la misión católica y el Estado colombiano 
que las facultaba para administrar y dirigir las escuelas públicas para varones, que posteriormente 1953 se extenderá a todos los territorios con presencia indígena, quedando la educación fuera del ámbito del conocimiento científico y permaneciendo en campo de lo teológico.

La educación indígena planteada desde el Estado, tenía una labor claramente evangelizadora, el objetivo destruir todo rasgo de la identidad cultural. Esta tendencia se fortaleció con la constitución de 1886 y su tendencia regeneradora

La Regeneración fue el movimiento que sentó las bases para la consolidación de la burguesía colombiana de acuerdo con Medina (1941). La organización nacional bajo una armonización de la libertad y el orden; del progreso y la estabilidad en función del capitalismo que empezaba a desarrollarse mediante la inversión y generaba ya acumulación de capital para las élites gobernantes. Este esquema expresaba la necesidad de garantías para los empresarios y sus inversiones.

La situación de los grupos indígenas en el contexto de las pugnas políticas que condujeron a la llamada regeneración y la expedición de la Constitución de 1886, inspirada en una ideología católica e hispanista, en la que el pasado colonial fue reivindicado y valorado positivamente, y se otorgó un rol especial a la religión católica como forjadora del orden de la nación. En este contexto, se expidió la ley 89 de 1890, que determinaba como debían ser gobernados los salvajes que se redujeran a la vida civilizada. Los indígenas quedaron al margen de la "legislación general" de la República, bajo la tutela de las misiones católicas, y fueron considerados como "menores de edad" en lo que atañe al régimen civil y penal de la Nación. En lo educativo propiamente dicho y bajo el Concordato, también se firmaron convenios para el desarrollo de proyectos misioneros en 1903 y 1928, mediante los cuales se reorganizaron Territorios nacionales y se definieron como zonas de Misiones con el propósito de entregarlos a las diversas órdenes religiosas 
católicas para Civilizar a los grupos indígenas que habitaban dichos territorios, la enseñanza se basaba en difundir la moral cristiana y la occidentalización de los indios (Pineda, 2016).

Este panorama educativo se extendió hasta la década de los 70 del siglo XX, cuando la comunidad arhuaca del departamento del Cesar presenta una propuesta en 1977, al Ministerio de Educación Nacional, sobre una educación bilingüe intercultural con el propósito de recuperar la tradición cultural. A partir de esta iniciativa se da origen al decreto 1142 de 1978 reglamentario del artículo 11 decreto ley 088 de 1976.

De igual manera, el Ministerio de Educación Nacional crea el grupo de etnoeducación para asesorar e impulsar programas de etnoeducación en las comunidades indígenas autorizando a la secretaría de educación del departamento del Cesar para aplicar el plan curricular o modelo educativo propuesto por la comunidad arhuaca. Asumir la orientación y administración de los servicios educativos de los establecimientos oficiales de la educación básica de la sierra Nevada de Santa Marta. Asegurar la participación de las comunidades indígenas, el ordenamiento y administración de estos programas estará dirigido por el Fondo Educativo Regional (FER) (Uribe, 1999, p. 4).

En la década los 80 se intensificaron las críticas y protestas contra el modelo educativo oficial, por parte de los grupos indígenas, el descontento se ilustra en el documento de Manuel Trino Morales, presentado durante en el Primer Seminario de Etnoeducación realizado en agosto de 1985, en Girardot, Cundinamarca:

[...] los indígenas creemos que la educación que el Estado ha venido imponiéndonos no es ajena a un propósito deliberado y planificado hacia el arrasamiento de nuestras culturas tradicionales con miras al logro de la mal llamada vinculación del indígena al desarrollo 
nacional y a integrarnos a la cultura dominante. Solo así se explica desde la Conquista hasta hoy la persecución y desconocimiento sistemático de nuestras formas tradicionales de educación, que forman al niño para defenderse frente a las necesidades que le demanda su medio, con una visión coherente y respetuosa de la naturaleza, que crea altos principios morales, guías de nuestra vida comunitaria; lo que es demostrable por la existencia actual de diferentes comunidades que en mayor o en menor grado conservamos elementos propios de nuestra cultura, testimonio de la dura lucha que venimos librando (Morales, 1995, p. 190).

Las normativas etnoeducativas que fueron inicialmente para los arhuacos del Cesar, se hacen universales para el contexto indígena colombiano con una serie de normas, decretos y resoluciones que propiciaron la participación activa de los indígenas en sus procesos educativos, y se abre la posibilidad para que los indígenas funcionen bajo un régimen especial diferente al sistema educativo nacional.

Al mismo tiempo se hacen excepciones para los centros educativos indígenas en la aplicación de programas educativos nacionales; los directivos docentes no están obligados a cumplir con el requisito de título profesional; se crea así mismo, el comité nacional de lingüística aborigen y se excluye del sistema de concursos y evaluación a los aspirantes a docentes para comunidades indígenas.

Concluye Uribe (1999, p. 4), que los procesos que los indígenas venían desarrollando en la creación de sus "proyectos educativos" partiendo de su realidad cultural se frenan, ya que el grupo de etnoeducación del Ministerio de Educación Nacional se convierte en doctrinero de la etnoeducación y etnodesarrollo, se interesa más en propagar las "etnoteorías", liderando las políticas y programas 
de educación del gobierno, desconociendo las experiencias de las comunidades.

En la constitución política de Colombia de 1991, se reconoce por primera vez la diversidad étnica y cultural de la nación colombiana, haciendo de los pueblos indígenas, afros y demás componentes de la diversidad cultural sujetos de derecho lo que significa darles vida jurídica y el compromiso de respetar y visibilizar los territorios, lenguas y dialectos, usos y costumbres de los grupos étnicos.

\section{Etnoeducación como Propuesta Educativa para los Grupos Étnicos}

En principio la etnoeducación surge como opción para los pueblos indígenas, como respuesta a las inconformidades y protestas al modelo educativo oficial. A partir del decreto 1142 de 1978 se van perfilando los cimientos de una etnoeducación para los pueblos indígenas que en principio se llamaba educación bilingüe intercultural con el fin de revitalizar la lengua, cultura y tradición de estos grupos.

El Estado colombiano emite la resolución 3454 de 1984 en la que se establecen los lineamientos generales de la educación indígena nacional; en su parte resolutiva, artículo 1, los programas para la educación formal y no formal de las comunidades indígenas del país se orientarán y desarrollarán de acuerdo con la guía o lineamientos formulados por el Ministerio de Educación Nacional, enmarcado dentro del enfoque definido como etnodesarrollo y su componente educativo denominado Etnoeducación.

Se define el etnodesarrollo

como el ejercicio de la capacidad social de decisión de un pueblo sobre el manejo de los recursos de su cultura para construir su futuro de acuerdo con un proyecto que 
se defina según sus propios valores y aspiraciones" y por Etnoeducación "un proceso social permanente inmerso en la cultura propia, que consiste en la adquisición de conocimientos y valores y en el desarrollo de habilidades y destrezas, que capacita a la persona para participar plenamente en el control cultural de su comunidad (Ministerio de Educación Nacional, resolución 3454 de 1984; Artículo 1).

Con el decreto 804 de 1995 se reglamenta la atención educativa para grupos étnicos dentro de la Ley 115 de 1994 o Ley General de la Educación, define la educación para grupos étnicos como un servicio público educativo que se construye con la participación colectiva. En el artículo 3 establece que en las entidades territoriales donde existan asentamientos de comunidades indígenas, negras o raizales se deberá incluir en los respectivos planes de desarrollo educativo propuestas de etnoeducación para atender esta población.

Estas disposiciones plantean la participación comunitaria de las etnias en la definición de sus procesos etnoeducativos y por primera vez se enuncia la presencia de las comunidades negras y raizales como partícipes de esta condición étnica.

Posteriormente se adopta el concepto de etnoeducación definido como lo concibe Agreda (1999, p. 260):

Los procesos etnoeducativos para los pueblos indigenas están estructurados teniendo en cuenta el territorio, cosmovisión, interculturalidad, usos y costumbres, sumándoles los principios de integralidad, diversidad lingüística y étnica, participación comunitaria, flexibilidad y progresividad. Para "desarrollar el programa de etnoeducación se han privilegiado cinco componentes, 
la formación y capacitación, profesionalización, nivelación y actualización, la investigación: lingüística, antropología, pedagogía y proyectos educativos comunitarios, diseño curricular: primaria y secundaria, producción de materiales en las regiones: pedagógico e informativo y publicaciones nacionales, seguimiento y evaluación".

La anterior definición presenta una percepción desde dentro debido a que es una líder indígena quien lo enuncia y promueve como opción que abarca las demandas fundamentales de las etnias indígenas.

Artunduaga (1997, p. 25), plantea que:

los procesos etnoeducativos deben hundir sus raíces en la cultura de cada pueblo, de acuerdo a los patrones y mecanismos de socialización de cada uno en particular, propiciando una articulación a través de una relación armónica entre lo propio y lo ajeno en la dimensión de interculturalidad.

Mientras Agreda plantea una visión muy cercana a la realidad étnica y cultural de los indígenas, Artunduaga hace una metáfora de lo que deben ser los procesos etnoeducativos en las comunidades indígenas coincidiendo con Agreda en la relación de la etnoeducación como proceso propio y orientado a salvaguardar los bienes más preciados de los grupos indígenas, territorio, lengua y cultura. 


\section{Resultados de la Investigación}

\section{La Etnoeducación como Programa Académico de la Universidad De La Guajira}

Elestablecimiento del programa de etnoeducación en launiversidad de La Guajira significó la puesta en escena de una serie de supuestos acerca de la interculturalidad, el potencial humano, la educabilidad, el reconocimiento de los valores de la etnia nativa, la posibilidad de administrar procesos educativos diferenciados, la profesionalización de docentes indígenas en todo el departamento guajiro. También significó el cambio de la orientación de los procesos educativos revalorando las tradiciones y saberes de los mayores como portadores de la cultura.

Dentro de este contexto se consideraba que:

Un programa de etnoeducación en el marco de una universidad regional, cumple una función social al tiempo que da respuesta, a las necesidades de capacitación del pueblo, por consiguiente, la revitalización de los patrones culturales de una etnia, a través de un programa de etnoeducación, tiene ciertas e importantes implicaciones en el descubrimiento científico y en las aplicaciones técnicas que elevan el nivel de vida del ser humano, sin desafiar el equilibrio que las etnias mismas han desarrollado y que garantizan su supervivencia. (Universidad de La Guajira, Documento Base. (Universidad de la Guajira, 1995, p. 23).

Consciente de su responsabilidad con la sociedad Guajira, la Universidad hace eco de las iniciativas que las organizaciones indígenas le proponen y asume el reto de concertar con varios estudiosos sociales un programa que responda a las necesidades de la comunidad y los capacite para sobrellevar el contraste cultural propio del departamento. 
En 1.995 la universidad de la guajira cristalizó el proyecto etnoeducativo universitario que:

ha sido uno de los pioneros de la etnoeducación en Colombia lo cual hace posible acercar más una realidad lejana con relación al propio sistema educativo, y la Universidad de La Guajira sería la herramienta para pulir todo un trabajo de autogestión de tecnificar los valores presentes en las comunidades y así demostrar la ruptura con unos lineamientos pedagógicos hegemónicos pretendidos "Universales". Una etnoeducación es, para los guajiros, el sistema que conduce a privilegiar la cultura y el pensamiento mismo, la de aceptar y compartir sistemas técnicos y de demostrar el espíritu de amor y compromiso con la sociedad.- Se pretende ir más allá del parámetro cotidiano para adquirir una retroalimentación a nivel de lo que se debería llamar etnodesarrollo. (Ibíd., p. 29).

Con estas ideas se da inicio a la construcción de la propuesta programática del programa de licenciatura en Etnoeducación y Proyecto Social que inicia labores en 1995 con dos modalidades, presencial y semipresencial.

Las disciplinas aportantes y énfasis aparecen hacia 1999, con el decreto 272 de 1998 que decía que el etnoeducador debía salir formado en un campo disciplinar, el título otorgado es de licenciado en etnoeducación para la educación media con énfasis en ciencias naturales o sociales.

Esta perspectiva especialista del saber de pregrado en etnoeducación entró en controversia con los modelos holísticos e integrales tradicionales de las sociedades con las que el programa trabajaba y produjo la separación del proyecto de varios docentes, sin 
embargo, el proceso continuó ya con menos vitalidad entre otras razones porque los grupos de estudiantes de las promociones más recientes eran jóvenes de origen urbano en su mayoría.

Este nuevo programa de Etnoeducación y proyecto Social en la Universidad de la Guajira, vino a convertirse en el pionero en toda la República de Colombia y profesionalizó, amparado en el Decreto 85 de 1980 a todos los profesores indígenas del departamento, legalizando su situación laboral dentro del intrincado escalafón del magisterio colombiano.

Periódicamente el programa entra en un periodo de reflexión y revisión y con ocasión de la actualización para el registro calificado, en 2010 se elabora un documento Maestro denominado Etnoeducación, interculturalidad y diversidad. Avalado por el Consejo Académico y aprobado por el Ministerio de Educación Nacional, con base en la ley 1188 de 2008. Este nuevo insumo está vigente para el programa de Etnoeducación.

La experiencia de trabajo reflexivo sobre la etnoeducación y la conciencia cobrada por las comunidades ante sus derechos fundamentales está propiciando la generación de procesos integradores entre los centros de pensamiento y las comunidades que con mayor claridad plantean sus demandas educativas y la aspiración de integrarlas a los requerimientos del Estado.

Cada vez se fue afianzando, gracias a los procesos etnoeducativos, la diferenciación entre lo indígena y lo campesino como realidades distintas dentro de las condiciones de marginalidad de la realidad latinoamericana.

Dentro de este contexto la población Wayuu inicia una nueva fase en la defensa territorial: 
la aparición de un elemento económico que no solo transformaba la región, sino que desplazaba y excluía de sus tierras a las comunidades wayuu, colocaba en La Guajira la discusión que en otros territorios nacionales se venía haciendo desde hacía algunas décadas. La explotación minera en gran parte del territorio trastornó la economía propia de las comunidades: las rozas de cultivo fueron abandonadas, el pastoreo restringido, al construirse la línea del tren, las zonas marítimas de pesca tradicional fueron ocupadas por el puerto, es decir se trastocan elementos económicos y culturales que recién 35 años después de iniciarse la explotación minera puede evaluarse como dañino para el Desarrollo de los wayuu, que no los ha beneficiado. Sus escuelas continúan siendo escasas, las enfermedades han proliferado, cada día les exigen más territorio, las prácticas corruptas han lesionado la unidad comunitaria que ha impedido que se organicen bajo un solo propósito de protesta. (Universidad de la Guajira, 1995, p. 39).

Se avizoran nuevos y futuros procesos producto de la relación renovada de comunidades y centros educativos para la búsqueda de modelos que reconozcan y exalten las dinámicas culturales propias, al tiempo que amplían las fronteras de interacción entre lo local étnico y lo nacional pluricultural.

\section{Conclusiones}

El desarrollo de los procesos etnoeducativos en la universidad de La Guajira ha significado una serie de transformaciones en los aspectos sociales relacionados con las luchas indígenas, los desarrollos 
universitarios y posteriormente la lucha de los afrodescendientes por su inclusión en las propuestas académicas nacionales.

En el caso colombiano, existe la propuesta de la etnoeducación como estrategia para ampliar la participación educativa de los grupos étnicos, que amplía la percepción inicial de considerar la etnoeducación como una educación para indígenas.

El desarrollo de la propuesta etnoeducativa como estrategia de participación en la multiculturalidad ha traído consigo una serie de nuevas realidades que obligan a repensar y reorganizar su propio desarrollo para atender los siguientes aspectos:

1. La diversidad cultural comprendida más allá de lo indígena por desconocimiento de otros componentes de la identidad nacional.

2. La etnoeducación como propuesta para grupos indígenas, como asegurar la articulación de esa diversidad indígena en visiones nacionales interétnicas.

3. La emergencia de las demandas afro como parte de la diversidad cultural y sus reclamos educativos.

En general, la etnoeducación en sus principios se consideró como una opción transformadora de la realidad educativa colombiana, sin embargo, con el transcurrir del tiempo ha pasado a ser un enfoque educativo que no cumplió con su rol complementario de formación del recurso humano en una nación pluriétnica y multicultural, segregándose a los espacios étnicos aún sin lograr una conjunción de esas visiones étnicas hacia una mirada étnica nacional. 


\section{Referencias}

Agreda,A. (1999). Culturas, lenguas, educación :memorias. EnD. Aguirre Lischt (Ed.), SimposiodeetnoeducaciónVIIICongreso Nacionaldeantropologia. Barranquilla, Colombia: Fondo de Publicaciones de la Universidad del Atlántico. Recuperado a partir de http://biblioteca.caroycuervo. gov.co/cgi-bin/koha/opac-detail.pl?biblionumber=75678\&query_ desc $=$ su $\% 3 \mathrm{~A} \% 22 \mathrm{ETNOEDUCACION} \% 22$

Ahern, E. J. G. (1991). El Desarrollo De La Educacion En Colombia 18201850. Universidad Pedagogica Nacional. Recuperado a partir de http://aulavirtual.iberoamericana.edu.co/recursosel/documentos_paradescarga/El desarrollo de la educación en Colombia (1).pdf

Artunduaga, L. A. (1997). La etnoeducación: una dimensión de trabajo para la educación en comunidades indígenas de Colombia. Revista Iberoamericana de Educación, (13), 35-45. Recuperado a partir de https://rieoei.org/historico/oeivirt/rie13a02.htm

Bodnar, Y. (2009). Una Mirada a La Etnoeducación Desde Las Prácticas Pedagógicas Culturales. En M. Rocha Vivas (Ed.), Interacciones Multiculturales, Los Estudiantes Indígenas en la Universidad (pp. 6786). Bogotá: Universidad Externado de Colombia. Recuperado a partir de https://s3.amazonaws.com/academia.edu.documents/31165598/ una_mirada_a_la_etnoeducacion.pdf?AWSAccessKeyId=AKIAIWOW YYGZ2Y53UL3A\&Expires=1525663481\&Signature=fVvew9baG5nG qsNQZSzV3zbI7ME\%3D\&response-content-disposition=inline\%3B filename\%3DUna_mirada_a_la_et

Burchardt, H. (2008). Desigualdad y democracia. Nueva sociedad, (215), 79-94. Recuperado a partir de http://dialnet.unirioja.es/servlet/ articulo?codigo $=3989177$

Gómez-Martínez, J. L. (2004). "La encrucijada del cambio: Simón Bolívar entre dos paradigmas" (una reflexión ante la encrucijada pos-industrial). Cuadernos Americanos, (104), 11-32. Recuperado a partir de https:// www.ensayistas.org/jlgomez/estudios/bolivar.htm

Habermas, J. (2009). La lucha por el reconocimiento en el Estado democráticos de derecho. En C. Taylor (Ed.), Multiculturalismo Y "La Política Del Reconocimiento " (p. 246). Fondo de Cultura Económica.

Krehoff, B. (2008). Multiculturalismo, indigenismo y derechos indígenas. En D. de la Fontaine \& P. C. Aparicio (Eds.), Diversidad cultural y desigualdad social en América latina y el Caribe: desafíos de la integración global. EDICIONES BÖLL. Recuperado a partir de 
http://www.iesalc.unesco.org.ve/dmdocuments/biblioteca/libros/83_ Diversidadculturalydesigualdadsocial.pdf\#page $=155$

Medina, A. (1941). Procesos del arte en Colombia Tomo I (1810-1930). Bogotá: Ediciones Uniandes.

Montoya, R. (2006). La cultura quechua hoy: Una conversación con Rodrigo Montoya. Hueso húmero, (49). Recuperado a partir de https://web. archive.org/web/20090319024551/http://huesohumero.perucultural. org.pe:80/textos/49/491.doc

Morales, M. T. (1985). Discurso del presidente de la Onic. En Memorias del Primer Seminario de Etnoeducación (25 al 31 de agosto). Bogotá: ONIC, MinEducación. Recuperado a partir de https://searchworks. stanford.edu/view/3417289

Pineda Camacho, R. (2016, octubre). ESTADO Y PUEBLOS INDÍGENAS EN EL SIGLO XIX. Revista Credencial. Recuperado a partir de http://www. revistacredencial.com/credencial/historia/temas/estado-y-pueblosindigenas-en-el-siglo-xix

Universidad de la Guajira. (1995). Documento Base. Programa de Etnoeducación y proyecto Social. 